08,04

\title{
Энергетическое обоснование образования димеров иттербия в монокристаллах форстерита
}

\author{
(C) В.Б. Дудникова ${ }^{1}$, Е.В. Жариков ${ }^{2}$, Д.А. Лис ${ }^{2}$, Н.Н. Еремин ${ }^{1}$ \\ ${ }^{1}$ Московский государственный университет им. М.В. Ломоносова, \\ Москва, Россия \\ ${ }^{2}$ Институт общей фризики им. А.М. Прохорова РАН, \\ Москва, Россия \\ E-mail: VDudnikova@hotmail.com
}

(Поступила в Редакцию 12 декабря 2018 г. В окончательной редакции 12 декабря 2018 г.

Принята к публикации 12 декабря 2018 г.)

\begin{abstract}
Проведено структурное компьютерное моделирование иттербийсодержаших кристаллов форстерита. Моделирование проводилось методом межатомных потенциалов с использованием программного комплекса GULP 4.1 (General Utility Lattice Program). Рассмотрены разные механизмы растворения иттербия в кристаллах форстерита, рассчитаны энергии растворения изолированных дефектов, а также заряженных и нейтральных кластеров различной конфигурации. Результаты расчета свидетельствуют о том, что образование кластеров иттербия с магниевой вакансией дает существенный выигрыш в энергии растворения. Образование нейтральных кластеров (димеров) в позиции $\mathrm{M1}:\left(\mathrm{Yb}_{\mathrm{Mg} 1} v_{\mathrm{Mg} 1} \mathrm{Yb}_{\mathrm{Mg} 1}\right)^{\times}$дает энергетический выигрыш в $1.7 \mathrm{eV}$ по сравнению со статистическим распределением дефектов. В результате моделирования показана энергетическая обусловленность образования димеров иттербия в кристаллах форстерита и предложена модель наиболее энергетически выгодного центра для позиции М1 - димера, состоящего из пары ионов трехвалентного иттербия с магниевой вакансией между ними, которые образуют цепочку, параллельную кристаллографической оси $c$.
\end{abstract}

Настоящая работа была выполнена при финансовой поддержке Российского научного фонда (грант № 18-12-00517) и Российского фонда фундаментальных исследований (грант № 18-07-01144 А).

DOI: 10.21883/FTT.2019.04.47422.339

\section{1. Введение}

Диэлектрические кристаллы с димерными центрами редкоземельных ионов представляют интерес для реализации устройств квантовой информатики. В этом отношении весьма интересными свойствами обладают кристаллы форстерита, легированного ионами иттербия [1]. В работе [1] методом стационарной и импульсной ЭПР-спектроскопии в $X$-диапазоне исследованы парамагнитные центры, образованные примесными ионами $\mathrm{Yb}^{3+}$ в синтетическом форстерите $\left(\mathrm{Mg}_{2} \mathrm{SiO}_{4}\right)$. Эти центры представляют собой одиночные ионы, замещающие магний в двух различных кристаллографических позициях, обозначаемых M1 и М2, и димерные ассоциаты, образованные двумя ионами $\mathrm{Yb}^{3+}$ в близлежащих позициях М1. Установлено, что имеется ярко выраженный механизм, благоприятствующий самоорганизации ионов иттербия в димерные ассоциаты. В настоящей работе подробно излагаются результаты структурного компьютерного моделирования для объяснения причин образования димерных центров в кристаллах $\mathrm{Mg}_{2} \mathrm{SiO}_{4}: \mathrm{Yb}$ и выбора наиболее энергетически выгодной конфигурации этих центров.

\section{2. Методика моделирования}

Моделирование кристаллов форстерита, в основу которого положена процедура минимизации энергии межатомных взаимодействий в структуре, было проведено с помощью программы GULP 4.1 (General Utility Lattice Program) [2]. Атомистический подход основан на использовании эмпирически определенных межатомных потенциалов, которые описывают взаимодействие между ионами в кристалле. Парный потенциал $U_{i j}$ взаимодействия ионов $i$ и $j$ с зарядами $q_{i}$ и $q_{j}$ является алгебраической суммой нескольких составляющих:

$$
U_{i j}\left(R_{i j}\right)=q_{i} q_{j} e^{2} / R_{i j}+A_{i j} \exp \left(-R_{i j} / \rho_{i j}\right)-c_{i j} / R_{i j}^{6} .
$$

Первый член представляет собой кулоновское взаимодействие, второй - отталкивание, возникающее при перекрывании электронных оболочек соседних атомов, а последний учитывает ван-дер-ваальсово взаимодействие, $R_{i j}$ - межатомное расстояние, $A_{i j}, \rho_{i j}, c_{i j}$ - параметры короткодействующих потенциалов, область действия которых в вычислениях составляла $12 \AA$.

Мы использовали формальные заряды ионов $\left(q_{\mathrm{Mg}}=+2 e, q_{\mathrm{Si}}=+4 e, q_{\mathrm{Yb}}=+3 e, q_{\mathrm{O}}=-2 e\right)$. Однако известно, что химические связи в $\mathrm{Mg}_{2} \mathrm{SiO}_{4}$ не могут быть адекватно описаны с помощью чисто ионной модели. Один из эффективных способов введения поправки 
на частичную ковалентность связей состоит в учете поляризуемости отдельных ионов, чаще всего анионов. Мы учитывали поляризуемость кислородных ионов $\mathrm{O}^{2-}$ с помощью так называемой „оболочечной модели“ [3]. В этом подходе ионы описываются как точечные положительно заряженные остовы, содержащие всю массу атома и окруженные отрицательно заряженной оболочкой, которая имитирует валентное электронное облако. Остов и оболочка связаны гармонической упругой константой $\chi_{i}$ :

$$
U_{i}^{s}=(1 / 2) \chi_{i} l_{i}^{2},
$$

где $l_{i}$ - расстояние между центрами остова и смещенной оболочки.

Трехчастичное взаимодействие $(i j k)$ ионов в связях $\mathrm{O}-\mathrm{Si}-\mathrm{O}$ в тетраэдрах $\mathrm{SiO}_{4}$ было учтено с помощью потенциала изгиба связи

$$
U_{i j k}=(1 / 2) \alpha_{i j k}\left(\theta_{i j k}-\theta_{o}\right)^{2} \text {, }
$$

где $\alpha_{i j k}$ - эмпирическая константа, $\theta_{i j k}$ - равновесный угол между связями, $\theta_{o}$ - угол в правильном тетраэдре $\left(109.47^{\circ}\right)$.

Использованные значения параметров потенциалов взаимодействия представлены в табл. 1. Для взаимодействия $\mathrm{Yb}-\mathrm{Os}$ они взяты из работы [4], для остальных параметров взаимодействия использовались позиционнозависимые значения параметров взаимодействия, полученные в работе [5].

Для оценки энергии дефектов в форстерите рассчитывалась энергия оптимизированной структурной модели кристалла. Энергия дефекта зависит от его взаимодействия с окружающей матрицей и определяется в результате минимизации статической энергии кристалла, содержащего дефект, при изменении положения атомов и дипольных моментов вокруг дефекта. Эта энергия представляет собой разницу между энергией кристалла, нарушенного (искаженного) дефектом, и энергией бездефектного кристалла.

Расчеты проводились по методике Мотта-Литтлтона [6], где вокруг дефекта выделяются две сферические области, с радиусами $R_{1}$ и $R_{2}, R_{1}<R_{2}$. В области $R_{1}$, непосредственно примыкающей к дефекту, энергия взаимодействия рассчитывается точно, путем решения уравнений для всех сил, действующих на каждый атом, с условием, чтобы их сумма была равна нулю. В промежуточной области между $R_{1}$ и $R_{2}$ энергия рассчитывается как функция смещений атомов в гармоническом приближении. В остальной части кристалла, которая рассматривается как диэлектрический континиум, оценивается поляризующее действие, обусловленное зарядом, расположенным в центре дефекта. В наших расчетах центральная область с радиусом $R_{1}=10 \AA$ включала более 600 атомов, следующая за ней сфера имела радиус $R_{2}=18 \AA$ и содержала более 3000 атомов.
Таблица 1. Параметры потенциалов межатомных взаимодействий в кристаллах $\mathrm{Mg}_{2} \mathrm{SiO}_{4}: \mathrm{Yb}\left(\mathrm{O}_{C}-\right.$ остов, $\mathrm{O}_{S}$ - валентная

\begin{tabular}{|c|c|c|}
\hline Связь & \multicolumn{2}{|c|}{ Параметры взаимодействия } \\
\hline \multirow{2}{*}{$\mathrm{Mg} 1-\mathrm{O} 1_{S}$} & $A, \mathrm{eV}$ & 1662 \\
\hline & $\rho, \AA$ & 0.295 \\
\hline \multirow{2}{*}{$\mathrm{Mg} 1-\mathrm{O} 2_{S}$} & $A, \mathrm{eV}$ & 1341 \\
\hline & $\rho, \AA$ & 0.295 \\
\hline \multirow{2}{*}{$\mathrm{Mg} 1-\mathrm{O} 3_{S}$} & $A, \mathrm{eV}$ & 1480 \\
\hline & $\rho, \AA$ & 0.295 \\
\hline \multirow{2}{*}{$\mathrm{Mg} 2-\mathrm{O} 1_{S}$} & $A, \mathrm{eV}$ & 1310 \\
\hline & $\rho, \AA$ & 0.295 \\
\hline \multirow{2}{*}{$\mathrm{Mg} 2-\mathrm{O} 2_{S}$} & $A, \mathrm{eV}$ & 1315 \\
\hline & $\rho, \AA$ & 0.295 \\
\hline \multirow{2}{*}{$\mathrm{Mg} 2-\mathrm{O}_{S}$} & $A, \mathrm{eV}$ & 1358 \\
\hline & $\rho, \AA$ & 0.295 \\
\hline \multirow{2}{*}{$\mathrm{Yb}-\mathrm{O}_{s}$} & $A, \mathrm{eV}$ & 1309.6 \\
\hline & $\rho, \AA$ & 0.3462 \\
\hline \multirow{3}{*}{$\mathrm{Si}-\mathrm{O} 1_{S}$} & $A, \mathrm{eV}$ & 1283.908 \\
\hline & $\rho, \AA$ & 0.3205 \\
\hline & $C, \mathrm{eV} \AA^{6}$ & 10.6616 \\
\hline \multirow{3}{*}{$\mathrm{Si}-\mathrm{O} 2_{S}$} & $A, \mathrm{eV}$ & 1280 \\
\hline & $\rho, \AA$ & 0.3205 \\
\hline & $C, \mathrm{eV} \AA^{6}$ & 10.6616 \\
\hline \multirow{3}{*}{$\mathrm{Si}-\mathrm{O} 3_{S}$} & $A, \mathrm{eV}$ & 1283.9073 \\
\hline & $\rho, \AA$ & 0.3205 \\
\hline & $C, \mathrm{eV} \AA^{6}$ & 10.6616 \\
\hline \multirow{3}{*}{$\mathrm{O}_{S}-\mathrm{O}_{S}$} & $A, \mathrm{eV}$ & 22764.3 \\
\hline & $\rho, \AA$ & 0.149 \\
\hline & $C, \mathrm{eV} \AA^{6}$ & 27.88 \\
\hline
\end{tabular}
оболочка иона кислорода)

\begin{tabular}{c|c|c}
\hline \multicolumn{3}{c}{ Оболочечная модель для атомов кислорода } \\
\hline $\mathrm{O}_{s}-\mathrm{O}_{C}$ & $\chi, \mathrm{eV} \mathrm{A}^{-2}$ & 74.9204 \\
\hline \multicolumn{3}{c}{ Трехчастичные взаимодействия } \\
\hline $\mathrm{O}_{S}-\mathrm{Si}-\mathrm{O}_{S}$ & $\alpha, \mathrm{eV} \mathrm{rad}^{-2}$ & 2.09724
\end{tabular}

\section{3. Результаты и их обсуждение}

Растворение примесей в кристалле можно рассмотреть с помощью реакций обмена между кристаллической фазой и расплавом, содержащим основной и примесный компоненты в форме оксидов. Иттербий 
находится в форстерите в трехвалентном состоянии, при этом, его вхождение в октаэдрические позиции структуры происходит по гетеровалентному механизму. При гетеровалентном замещении магния трехвалентным иттербием образуется примесный дефект с избыточным положительным зарядом, который необходимо скомпенсировать. При отсутствии специально вводимых компенсаторов замещение на два трехвалентных иона иттербия происходит за счет удаления трех двухвалентных ионов магния с образованием одной магниевой вакансии. При этом точечные дефекты (примесные ионы иттербия и магниевая вакансия) могут распределяться статистически, а также образовывать заряженные или нейтральные кластеры.

При статистическом распределении дефектов процесс гетеровалентного замещения может быть представлен следующим образом

$$
\frac{1}{2} \mathrm{Yb}_{2} \mathrm{O}_{3}+\frac{3}{2} \mathrm{Mg}_{\mathrm{Mg}}^{\times} \Leftrightarrow \mathrm{Yb}_{\mathrm{Mg}}^{\bullet}+\frac{1}{2} v_{\mathrm{Mg}}^{\prime \prime}+\frac{3}{2} \mathrm{MgO} .^{1}
$$

Энергия этого процесса в расчете на один ион иттербия может быть получена из соотношения

$E_{S}=\frac{1}{2} E_{d}\left(2 \mathrm{Yb}_{\mathrm{Mg}}^{\bullet}+v_{\mathrm{Mg}}^{\prime \prime}\right)+\frac{3}{2} E_{\text {str. }}(\mathrm{MgO})-\frac{1}{2} E_{\text {str. }}\left(\mathrm{Yb}_{2} \mathrm{O}_{3}\right)$

где $E_{d}$ - энергии соответствующих дефектов в форстерите, при этом невзаимодействующие дефекты (находящиеся в скобках) будем обозначать отделенными друг от друга знаком плюс. $E_{s t r .}$ - энергии решетки оксидов, которые по результатам расчета составляют -41.3 и $-136.8 \mathrm{eV}$ для $\mathrm{MgO}$ и $\mathrm{Yb}_{2} \mathrm{O}_{3}$ соответственно. Результаты расчета энергии одиночных дефектов, участвующих в процессе растворения иттербия и энергии растворения иттербия в расчете на один ион при статистическом распределении дефектов представлены в табл. 2.

Из табл. 2 видно, что образование вакансии магния в позиции М1, выгоднее, чем в позиции М2. Этот вывод подтверждают также квантово-механические оценки [8]. Согласно нашим расчетным данным, одиночный дефект иттербия в позиции М2 энергетически более выгоден, чем в позиции М1. Наименьшая энергия растворения изолированных дефектов составляет $2 \mathrm{eV}$, что соответствует случаю, когда ион иттербия локализуется в позиции М2, а магниевая вакансия - в позиции М1 (см. табл. 2).

Разноименно заряженные дефекты притягиваются и могут образовывать кластеры. Влияние кластеризации рассмотрим на примере ионов иттербия, локализованных в позиции M1, кластеры (димеры) которых были обнаружены, как уже упоминалось выше, при ЭПР исследовании этих кристаллов. В случае, когда ион иттербия и магниевая вакансия располагаются рядом,

\footnotetext{
1 Здесь и далее приняты обозначения, предложенные Крегером [7]: нижний индекс соответствует позиции дефекта в кристалле, верхний - эффективному избыточному положительному $(\bullet)$, избыточному отрицательному $\left(^{\prime}\right)$ или нейтральному $\left({ }^{\times}\right)$заряду по отношению к заряду соответствующей позиции в матрице, $v$ - вакансия.
}

Таблица 2. Энергии дефектов $\left(E_{d}\right)$ и энергии растворения $\left(E_{S}\right)$ иттербия в виде изолированных дефектов в кристаллах $\mathrm{Mg}_{2} \mathrm{SiO}_{4}: \mathrm{Yb}$

\begin{tabular}{c|c|c}
\hline Дефекты & $E_{d}, \mathrm{eV}$ & $E_{S}, \mathrm{eV}$ \\
\hline$v_{\mathrm{Mg} 1}^{\prime \prime}$ & 24.1 & \\
\hline$v_{\mathrm{Mg} 2}^{\prime \prime}$ & 26.4 & \\
\hline $\mathrm{Yb}_{\mathrm{Mg} 1}^{\bullet}$ & -16.0 & \\
\hline $\mathrm{Yb}_{\mathrm{Mg} 2}^{\bullet}$ & -16.5 & \\
\hline $\mathrm{Yb}_{\mathrm{Mg} 1}^{\bullet}+\frac{1}{2} v_{\mathrm{Mg} 1}^{\prime \prime}$ & & 2.5 \\
\hline $\mathrm{Yb}_{\mathrm{Mg} 1}^{\bullet}+\frac{1}{2} v_{\mathrm{Mg} 2}^{\prime \prime}$ & & 3.6 \\
\hline $\mathrm{Yb}_{\mathrm{Mg} 2}^{\bullet}+\frac{1}{2} v_{\mathrm{Mg} 1}^{\prime \prime}$ & & 2.0 \\
\hline $\mathrm{Yb}_{\mathrm{Mg} 2}^{\bullet}+\frac{1}{2} v_{\mathrm{Mg} 2}^{\prime \prime}$ & & 3.2
\end{tabular}

может образовываться заряженный кластер, который имеет отрицательный заряд по сравнению с ненарушенной решеткой. Этот заряд может быть скомпенсирован не связанным в кластер удаленным вторым ионом иттербия. Энергия растворения для этого процесса может быть рассчитана следующим образом:

$$
\begin{aligned}
E_{S}= & \frac{1}{2} E_{d}\left[\left(\mathrm{Yb}_{\mathrm{Mg} 1} v_{\mathrm{Mg} 1}\right)^{\prime}+\mathrm{Yb}_{\mathrm{Mg} 1}^{\bullet}\right]+\frac{3}{2} E_{\text {str. }}(\mathrm{MgO}) \\
& -\frac{1}{2} E_{\text {str. }}\left(\mathrm{Yb}_{2} \mathrm{O}_{3}\right)
\end{aligned}
$$

(При обозначении кластеров рядом расположенные дефекты заключены в круглые скобки с указанием результирующего заряда кластера

Магниевая вакансия может также располагаться рядом с двумя ионами иттербия, образуя в этом случае нейтральный кластер (димер). Энергия растворения этого кластера

$$
\begin{aligned}
E_{S}= & \frac{1}{2} E_{d}\left(\mathrm{Yb}_{\mathrm{Mg} 1} v_{\mathrm{Mg} 1} \mathrm{Yb}_{\mathrm{Mg} 1}\right)^{\times}+\frac{3}{2} E_{\text {str. }}(\mathrm{MgO}) \\
& -\frac{1}{2} E_{\text {str. }}\left(\mathrm{Yb}_{2} \mathrm{O}_{3}\right)
\end{aligned}
$$

Структуры разных кластеров ионов иттербия, занимающих позицию M1, с магниевой вакансией представлены на рисунке. В левой колонке рисунка представлены заряженные ассоциаты, а в правой - нейтральные ассоциаты (димеры). Энергии заряженных и нейтральных кластеров различной конфигурации, энергии ассоциации в этих кластерах, представляющие собой разницу между энергией кластера и энергией соответствующих изолированных дефектов, а также энергии растворения иттербия в виде кластера в расчете на один ион представлены в табл. 3. За счет энергии ассоциации при образовании заряженных кластеров энергия кристалла уменьшается на 1.9 и $0.6 \mathrm{eV}$ при локализации вакансии магния в 


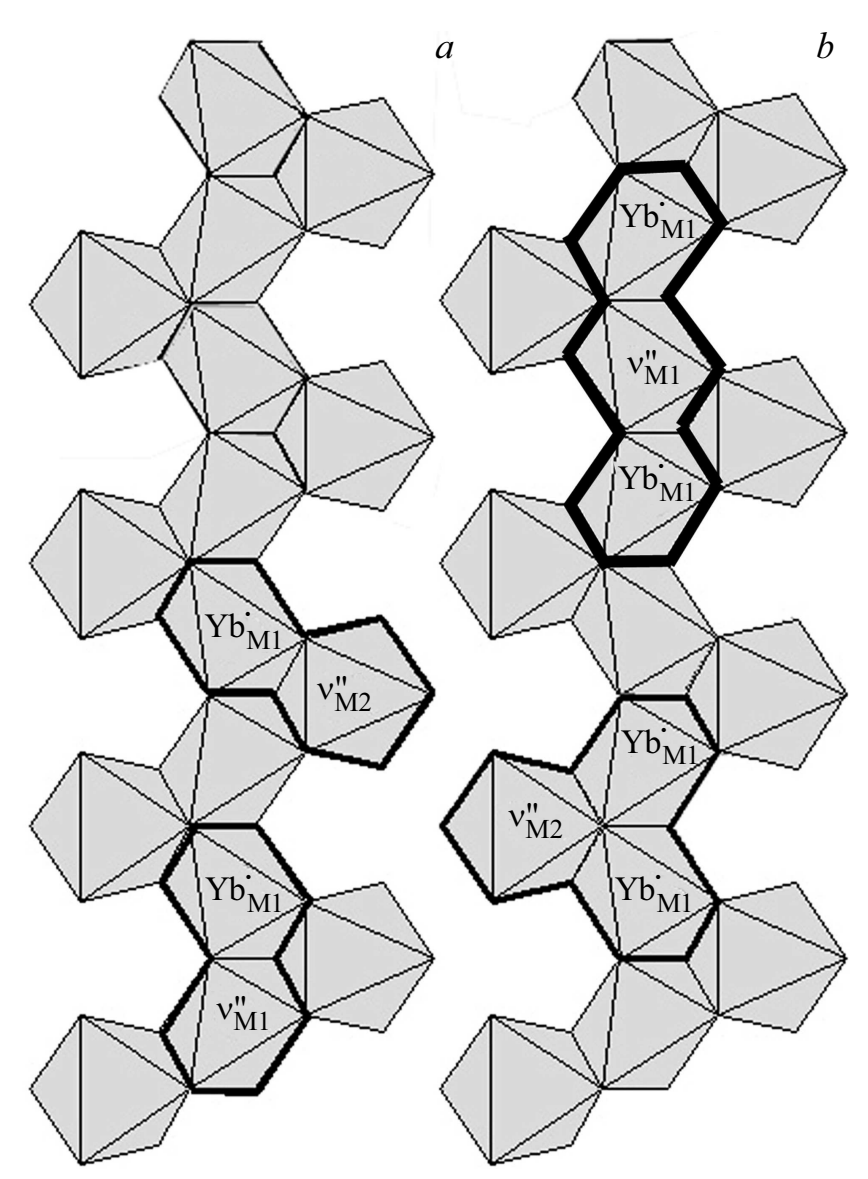

Структура кластеров иттербия $\mathrm{Yb}_{\mathrm{Mg} 1}$ в форстерите. (a) заряженные ассоциаты, $(b)$ - нейтральные ассоциаты-димеры.

позиции М1 и М2 соответственно. В то же время, образование нейтральных димеров понижает энергию кристалла на 3.4 и $3.3 \mathrm{eV}$ для случаев вакансии магния в M1 и М2. Что демонстрирует энергетическую выгодность образования ассоциатов иттербия в кристаллах форстерита.

Таблица 3. Энергии дефектов $\left(E_{d}\right)$, энергии ассоциации в кластерах $\left(E_{\text {as }}\right)$ и энергии растворения ионов $\mathrm{Yb}^{3+}$ в виде кластеров $\left(E_{S}\right)$ (в расчете на один ион иттербия в кристаллах $\left.\mathrm{Mg}_{2} \mathrm{SiO}_{4}: \mathrm{Yb}\right)$

\begin{tabular}{c|r|r|c}
\hline Дефекты & $E_{d}, \mathrm{eV}$ & $E_{\mathrm{as}}, \mathrm{eV}$ & $E_{S}, \mathrm{eV}$ \\
\hline$\left(v_{\mathrm{Mg} 1} \mathrm{Yb}_{\mathrm{Mg} 1}\right)^{\prime}$ & 6.2 & 1.9 & \\
\hline$\left(v_{\mathrm{Mg} 2} \mathrm{Yb}_{\mathrm{Mg} 1}\right)^{\prime}$ & 9.8 & 0.6 & \\
\hline$\left(\mathrm{Yb}_{\mathrm{Mg} 1} v_{\mathrm{Mg} 1} \mathrm{Yb}_{\mathrm{Mg} 1}\right)^{\times}$ & -11.3 & 3.4 & \\
\hline$\left(\mathrm{Yb}_{\mathrm{Mg} 1} v_{\mathrm{Mg} 2} \mathrm{Yb}_{\mathrm{Mg} 1}\right)^{\times}$ & -8.9 & 3.3 & \\
\hline$\frac{1}{2}\left[\left(v_{\mathrm{Mg} 1} \mathrm{Yb}_{\mathrm{Mg} 1}\right)^{\prime}+\mathrm{Yb}_{\mathrm{Mg} 1}^{\bullet}\right]$ & & & 1.6 \\
\hline$\frac{1}{2}\left[\left(v_{\mathrm{Mg} 2} \mathrm{Yb}_{\mathrm{Mg} 1}\right)^{\prime}+\mathrm{Yb}_{\mathrm{Mg} 1}^{\bullet}\right]$ & & & 3.4 \\
\hline$\frac{1}{2}\left(\mathrm{Yb}_{\mathrm{Mg} 1} v_{\mathrm{Mg} 1} \mathrm{Yb}_{\mathrm{Mg} 1}\right)^{\times}$ & & & 0.8 \\
\hline$\frac{1}{2}\left(\mathrm{Yb}_{\mathrm{Mg} 1} v_{\mathrm{Mg} 2} \mathrm{Yb}_{\mathrm{Mg} 1}\right)^{\times}$ & & & 2.0
\end{tabular}

Результаты расчета свидетельствуют о том, что образование кластеров иттербия с магниевой вакансией дает существенный выигрыш в энергии растворения. Кластеры, в которых вакансия магния занимает позицию М2, менее энергетически выгодны по сравнению с теми, где вакансия локализована в позиции М1.

Таким образом, наиболее энергетически выгодно образование нейтральных кластеров (димеров) $\left(\mathrm{Yb}_{\mathrm{Mg} 1} v_{\mathrm{Mg} 1} \mathrm{Yb}_{\mathrm{Mg} 1}\right)^{\times}$. Димеры $\left(\mathrm{Yb}_{\mathrm{Mg} 1} v_{\mathrm{Mg} 1} \mathrm{Yb}_{\mathrm{Mg} 1}\right)^{\times}$на рисунке, выделены жирными линиями. Если ионы иттербия и компенсирующая магниевая вакансия являются изолированными дефектами (статистическое распределение), то энергия растворения в расчете на один ион иттербия составляет $2.5 \mathrm{eV}$ (см. табл. 2). При образовании димера (локальная компенсация заряда) энергия растворения иттербия вместе с дефектом, компенсирующим электронейтральность, составляет $0.8 \mathrm{eV}$ (в расчете на один ион иттербия), т.е. уменьшается на $1.7 \mathrm{eV}$ по сравнению с их статистическим распределением.

\section{4. Заключение}

С использованием позиционно-зависимых потенциалов межатомного взаимодействия проведено атомистическое моделирование кристаллов форстерита, легированного ионами иттербия. Рассчитаны энергии растворения изолированных дефектов, а также заряженных и нейтральных кластеров различной конфигурации. В результате моделирования показана энергетическая обусловленность образования димеров иттербия в кристаллах форстерита и обоснована модель наиболее энергетически выгодного центра иттербия в позиции М1 - димера, состоящего из пары ионов трехвалентного иттербия с магниевой вакансией между ними, которые замещают три магниевых иона образуя цепочку, параллельную кристаллографической оси $c$.

Работа выполнена с использованием оборудования Центра коллективного пользования сверхвысокопроизводительными вычислительными ресурсами МГУ им. М.В. Ломоносова

\section{Список литературы}

[1] В.Ф. Тарасов, А.А. Суханов, В.Б. Дудникова, Е.В. Жариков, Д.А. Лис, К.А. Субботин. Письма в ЖЭТФ 106, 2, 78 (2017).

[2] J. Gale, A.L. Rohl. Mol. Simul. 29, 5, 291 (2003).

[3] B.G. Dick, A.W. Overhauser. Phys. Rev. 112, 90 (1958).

[4] G.V. Lewis, C.R.A. Catlow. J. Phys C 18, 1149 (1985).

[5] В.Б. Дудникова, В.С. Урусов, Е.В. Жариков. ФТТ 56, 1327 (2014).

[6] N.F. Mott, M.J. Littleton. Trans. Faraday Soc. 34, 485 (1938).

[7] Ф. Крегер. Химия несовершенных кристаллов. Мир, М. (1969). $654 \mathrm{c}$.

[8] J. Brodholt. Am. Mineralogist 82, 1049 (1997).

Редактор Т.Н. Василевская 